\title{
GENERATION OF SURFACE WAVES DUE TO INITIAL AXISYMMETRIC SURFACE DISTURBANCE IN WATER WITH A POROUS BOTTOM
}

\author{
P. KUNDU \\ Department of Mathematics, Jadavpur University \\ Kolkata-700032, INDIA \\ E-mail:kundupiyali92@gmail.com \\ B.N. MANDAL ${ }^{*}$ \\ Physics and Applied Mathematics Unit, Indian Statistical Institute \\ 203 B.T Road, Kolkata-700108, INDIA \\ E-mail: bnm2006@rediffmail.com
}

\begin{abstract}
A two-dimensional Cauchy Poisson problem for water with a porous bottom generated by an axisymmetric initial surface disturbance is investigated here. The problem is formulated as an initial value problem for the velocity potential describing the motion in the fluid. The Laplace and Hankel transform techniques have been used in the mathematical analysis to obtain the form of the free surface in terms of a multiple infinite integral. This integral is then evaluated asymptotically by the method of stationary phase. The asymptotic form of the free surface is depicted graphically in a number of figures for different values of the porosity parameter and for different types of initial disturbances.
\end{abstract}

Key words: axisymmetric surface disturbance, porous bottom, Laplace and Hankel transform, method of stationary phase, free surface depression.

\section{Introduction}

The problem of generation of surface waves due to an explosion above or within the water can be formulated as an initial value problem assuming linear theory of water waves. The explosion may occur above or below the ocean surface. When the explosion occurs within the water the initial condition is taken as an initial displacement (elevation or depression) distributed over a certain region of the free surface. However when the explosion occurs above water, the initial condition can be considered as an initial impulsive pressure distributed over a certain region of the free surface. Due to these types of initial disturbances, problems of wave generation in deep water were studied by Lamb [1] and Stoker [2]. For axisymmetric initial disturbance concentrated at a point on the free surface, the problem of generation of surface waves in deep water was studied by Stoker [2]. The form of the free surface elevation was obtained in terms of a multiple infinite integral using the Hankel transform and the asymptotic form of the free surface was found by using the stationary phase method twice.

For the three dimensional unsteady motion, the axially symmetric disturbance was considered by Kranzer and Keller [4] in finite depth water and they compared the theoretical result with experimental results. They also considered different types of impulse distribution to analyze the nature of the free surface elevation and the associated phenomenon of surface waves. Mandal and Mukherjee [5] considered wave generation at an inertial surface due to an axisymmetric initial surface disturbance. Maiti and Mandal [6] considered water waves generated due to initial axisymmetric disturbances in deep water covered by a thin layer of ice in the upper surface.

\footnotetext{
* To whom correspondence should be addressed
} 
In all these problems, the ocean bottom is either considered rigid or of infinite depth. However, in reality the ocean bottom is actually porous. In the present problem, we consider the generation of surface waves due to different types of initial axisymmetric disturbances at the free surface in finite depth water with a porous bottom. Here a special type of porous bottom is considered as in Martha et al. [7] and Maiti, and Mandal [8] where the porosity parameter is described by a real quantity $G$ whose dimension is taken as (length) ${ }^{-1}$.

Three types of initial disturbances have been considered in this paper. In the first case, an initial axially symmetric displacement concentrated at a point on the free surface has been considered and in the second case it is an impulse concentrated at the origin while in the third case, a parabolic impulse distribution at the free surface has been considered as in Kranzer and Keller [4]. The problem is formulated as an initial value problem assuming linear theory. Laplace and Hankel transform techniques are used to solve the problem. Finally the depression of the free surface at any time $t$ is obtained as a multiple infinite integral. For the first two disturbances the integral is evaluated asymptotically for large time and distance using the stationary phase method twice. However, for the case of parabolic impulse distribution, the multiple integral is evaluated by the method of steepest-descent as in Jeffreys and Lapwood [3]. The asymptotic form of the free surface is depicted graphically against a non-dimensional distance for fixed time and against nondimensional time for a fixed distance and for different values of non-dimensionalised porosity parameter.

\section{Mathematical formulation}

A cylindrical co-ordinate system $(r, \theta, y)$ is chosen in which the $y$-axis is taken vertically downwards in the fluid region and $y=h$ corresponds to the bottom composed of some specific kind of porous materials. The porous bottom is characterized by a real quantity $G$ which has the dimension of inverse of length. The fluid is assumed to be inviscid and the motion in the fluid starts from rest so that it is irrotational and the motion is described by a velocity potential $\varphi(r, y, t)$ satisfying the Laplace equation

$$
\frac{1}{r} \frac{\partial}{\partial r}\left(r \frac{\partial \varphi}{\partial \mathrm{r}}\right)+\frac{\partial^{2} \varphi}{\partial y^{2}}=0, \quad y \geq 0, \quad t \geq 0
$$

the boundary conditions

$$
\begin{aligned}
& \frac{\partial^{2} \varphi}{\partial t^{2}}-g \frac{\partial \varphi}{\partial y}=0 \text { on } y=0, \\
& \frac{\partial \varphi}{\partial y}-G \varphi=0 \text { on } y=h
\end{aligned}
$$

where $G$ is the porosity parameter of the fluid bottom. The initial conditions are

$$
\varphi(r, 0,0)=0, \quad \varphi_{t}(r, 0,0)=g G(r),
$$

when the initial axially symmetric depression $G(r)$ of the free surface at a distance $r$ from the origin is prescribed and $g$ is the acceleration due to gravity, or

$$
\varphi(r, 0,0)=-\frac{F(r)}{\rho}, \quad \varphi_{t}(r, 0,0)=0,
$$

when an initial axially symmetric impulse $F(r)$ is applied per unit area of the free surface at a distance $r$ from the origin, and $\rho$ is the density of water. 
The expression for free surface depression is given by

$$
\eta(r, t)=\frac{1}{g} \varphi_{t}(r, 0, t) .
$$

Introducing a characteristic length $l$, characteristic time $\sqrt{\frac{l}{g}}$, and characteristic mass $m$, we define the dimensionless quantities

$$
\bar{r}=\frac{r}{l}, \quad \bar{y}=\frac{y}{l}, \quad \bar{t}=t \sqrt{\frac{l}{g}}, \quad \bar{\eta}=\frac{\eta}{l}, \quad \bar{h}=\frac{h}{l}, \quad \bar{\varphi}=\frac{\varphi}{l \sqrt{g l}}, \quad \bar{G}=G l, \quad \bar{F}=\frac{l F}{m \sqrt{\frac{l}{g}}}, \quad \bar{\rho}=\frac{\rho l^{3}}{m} .
$$

Removing the bars the dimensionless quantities satisfy

$$
\begin{aligned}
& \frac{1}{r} \frac{\partial}{\partial r}\left(r \frac{\partial \varphi}{\partial r}\right)+\frac{\partial^{2} \varphi}{\partial y^{2}}=0, \quad y \geq 0, \quad t \geq 0, \\
& \frac{\partial^{2} \varphi}{\partial t^{2}}-g \frac{\partial \varphi}{\partial y}=0 \quad \text { on } \quad y=0, \\
& \frac{\partial \varphi}{\partial y}-G \varphi=0 \quad \text { on } \quad y=h .
\end{aligned}
$$

Initial conditions at the free surface are

$$
\begin{aligned}
& \varphi(r, 0,0)=0, \\
& \varphi_{t}(r, 0,0)=g G(r),
\end{aligned}
$$

or

$$
\varphi(r, 0,0)=-\frac{F(r)}{\rho},
$$

and

$$
\varphi_{t}(r, 0,0)=0
$$

according as the initial disturbance is axially symmetric depression of the free surface or an axially symmetric impulse at the free surface and the corresponding non dimensional depression of the free surface is to be obtained from the relation

$$
\eta(r, t)=\frac{1}{g} \varphi_{t}(r, 0, t)
$$

\section{Method of solution}

The Laplace and Hankel transform techniques are used to solve the above initial value problem. Let $\varphi(r, y, p)$ be the Laplace transform of $\varphi(r, y, t)$ at time $t$, defined as 


$$
\bar{\varphi}(r, y, p)=\int_{0}^{\infty} \varphi(r, y, t) e^{-p t} d t
$$

Taking the Laplace transform of the Eqs (2.1), (2.2), (2.3) and using the initial conditions (2.4), and (2.5) we have

$$
\begin{aligned}
& \frac{1}{r} \frac{\partial}{\partial r}\left(r \frac{\partial \bar{\varphi}}{\partial r}\right)+\frac{\partial^{2} \bar{\varphi}}{\partial y^{2}}=0, \quad y \geq 0, \\
& p^{2} \bar{\varphi}-\bar{\varphi}_{y}=G(r) \quad \text { on } \quad y=0, \\
& \bar{\varphi}_{y}-G \bar{\varphi}=0 \quad \text { on } \quad y=h .
\end{aligned}
$$

Let $\Psi(k, y, p)$ be the Hankel Transform of $\bar{\varphi}(r, y, p)$ defined by

$$
\Psi(k, y, p)=\int_{0}^{\infty} r \bar{\varphi}(r, y, p) J_{0}(k r) d r, \quad k>0 .
$$

Then $\Psi(k, y, p)$ satisfies

$$
\begin{aligned}
& \frac{d^{2} \Psi}{d y^{2}}-k^{2} \Psi=0, \quad k>0, \\
& p^{2} \Psi-\Psi_{y}=\hat{G}(k) \quad \text { on } \quad y=0, \\
& \Psi_{y}-G \Psi=0 \quad \text { on } \quad y=h
\end{aligned}
$$

where $\hat{G}(k)$ denotes the Hankel transform of $G(r)$.

The solution of Eq.(3.4) satisfying Eqs (3.5) and (3.6) can be written as

$$
\Psi(k, y, p)=\frac{\hat{G}(k)}{\mu(k)}[k \cosh k(y-h)+G \sinh k(y-h)]
$$

where

$$
\mu(k)=p^{2}(k \cosh k h-G \sinh k h)+k(k \sinh k h-G \cosh k h) .
$$

Taking the inverse Hankel transform

$$
\bar{\varphi}(r, y, p)=\int_{0}^{\infty} \frac{k \cosh k(y-h)+G \sinh k(y-h)}{\left(p^{2}+\Omega^{2}\right)(k \cosh k h-G \sinh k h)} \hat{G}(k) k J_{0}(k r) d k
$$

where

$$
\Omega^{2}(k)=\frac{k(k \tanh k h-G)}{(k-G \tanh k h)} .
$$

Now $\Omega^{2}(k)$ behaves differently for $G h<1$ and $G h>1$. 
Case A $(\boldsymbol{G h}<1)$ :

The graph of $\Omega^{2}(k)$ is shown in Fig.1 for $G h<1$. It is observed that there is a zero of $\Omega^{2}(k)$ at $k=\lambda_{1}$ and $\Omega^{2}(k)>0$ for $\lambda_{1} \leq k<\infty$ and $\Omega^{2}(k)<0$ for $0<k<\lambda_{1}$.

Let, $\Omega^{2}(k)=\left\{\begin{array}{ccc}-\mu_{1}^{2}(k) & \text { for } & 0<k<\lambda_{1} \\ \mu_{2}^{2}(k) & \text { for } & \lambda_{1} \leq k<\infty\end{array}\right.$

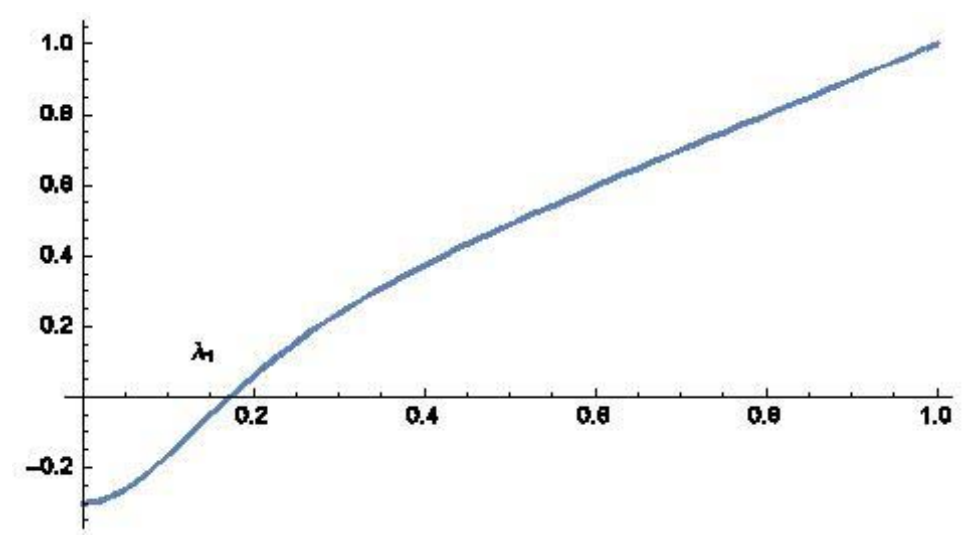

Fig.1. Graph of $\Omega^{2}(k)$ for $G h<1$.

Then

$$
\begin{aligned}
& \bar{\varphi}(r, y, p)=\int_{0}^{\lambda_{l}} \frac{k \cosh k(y-h)+G \sinh k(y-h)}{\left(p^{2}-\mu_{l}^{2}\right)(k \cosh k h-G \sinh k h)} \hat{G}(k) k J_{0}(k r) d k+ \\
& +\int_{\lambda_{1}}^{\infty} \frac{k \cosh k(y-h)+G \sinh k(y-h)}{\left(p^{2}+\mu_{2}^{2}\right)(k \cosh k h-G \sinh k h)} \hat{G}(k) k J_{0}(k r) d k .
\end{aligned}
$$

Taking the Laplace inversion we obtain

$$
\begin{aligned}
& \varphi(r, y, t)=\int_{0}^{\lambda_{l}} \frac{\sinh \left(\mu_{1} t\right)(k \cosh k(y-h)+G \sinh k(y-h)}{\mu_{l}(k \cosh k h-G \sinh k h)} \hat{G}(k) k J_{0}(k r) d k+ \\
& +\int_{\lambda_{1}}^{\infty} \frac{\sin \left(\mu_{2} t\right)(k \cosh k(y-h)+G \sinh k(y-h))}{\mu_{2}(k \cosh k h-G \sinh k h)} \hat{G}(k) k J_{0}(k r) d k .
\end{aligned}
$$

Free surface depression in the case of initial axially symmetric depression is given by

$$
\eta_{l}(r, t)=\int_{0}^{\lambda_{l}} \cosh \left(\mu_{1} t\right) \hat{G}(k) k J_{0}(k r) d k+\int_{\lambda_{1}}^{\infty} \cos \left(\mu_{2} t\right) \hat{G}(k) k J_{0}(k r) d k .
$$

In the case of axially symmetric impulse at the free surface the expression for depression of the free surface at time $t$ is obtained by a similar procedure as

$$
\eta_{2}(r, t)=-\frac{1}{\rho} \int_{0}^{\lambda_{1}} \mu_{1} \sinh \left(\mu_{1} t\right) \hat{F}(k) k J_{0}(k r) d k+\frac{1}{\rho} \int_{\lambda_{1}}^{\infty} \mu_{2} \sin \left(\mu_{2} t\right) \hat{F}(k) k J_{0}(k r) d k .
$$


Case B $(\boldsymbol{G h}>1)$ :

For $G h>1$ the numerator and denominator of $\Omega^{2}(k)$ are as shown in Fig.2 and $\Omega^{2}(k)$ in Fig.3. Let $k=\lambda_{3}$ be the positive zero of numerator $k(k \tanh (k h)-G)$ and $k=\lambda_{2}$ be the positive zero of denominator $(k-G \tanh (k h))$.

Then $\Omega^{2}(k)$ can be written as

$$
\Omega^{2}(k)=\left\{\begin{array}{ccc}
v_{1}^{2}(k) & \text { for } & 0 \leq k<\lambda_{2}-\varepsilon \\
-v_{2}^{2}(k) & \text { for } & \lambda_{2}+\varepsilon<k \leq \lambda_{3} . \\
v_{3}^{2}(k) & \text { for } & \lambda_{3}<k<\infty
\end{array}\right.
$$
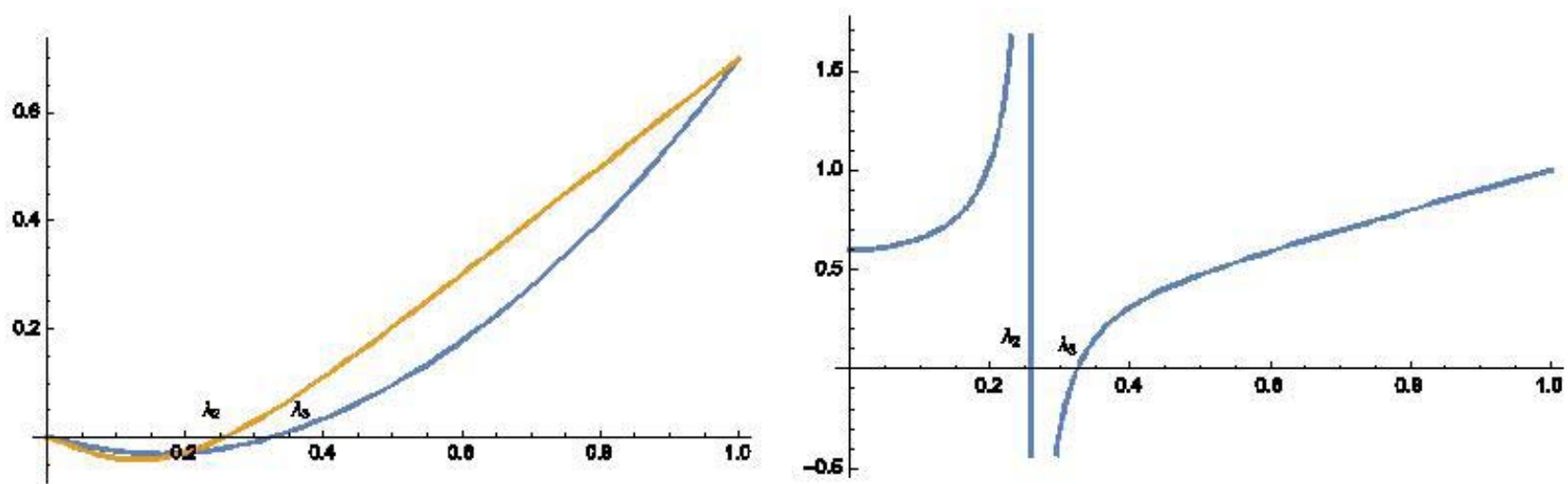

Fig.2. $k(k \tanh k h-G)$ and $(k-G \tanh (k h))$ for $G h>1$.

Fig.3. $\Omega^{2}(k)$ for $G h>1$.

where $\varepsilon$ is an arbitrary small positive real number. Then $\bar{\varphi}(r, y, p)$ can be written as

$$
\begin{aligned}
& \bar{\varphi}(r, y, p)=\lim _{\varepsilon \rightarrow 0}\left[\int_{0}^{\lambda_{2}-\varepsilon} \frac{k \cosh k(y-h)+G \sinh k(y-h)}{\left(p^{2}+v_{1}^{2}\right)(k \cosh k h-G \sinh k h)} \hat{G}(k) k J_{0}(k r) d k+\right. \\
& +\int_{\lambda_{2}+\varepsilon}^{\lambda_{3}} \frac{k \cosh k(y-h)+G \sinh k(y-h)}{\left(p^{2}-v_{2}^{2}\right)(k \cosh k h-G \sinh k h)} \hat{G}(k) k J_{0}(k r) d k+ \\
& \left.+\int_{\lambda_{3}}^{\infty} \frac{k \cosh k(y-h)+G \sinh k(y-h)}{\left(p^{2}+v_{3}^{2}\right)(k \cosh k h-G \sinh k h)} \hat{G}(k) k J_{0}(k r) d k\right] .
\end{aligned}
$$

Taking the Laplace inversion, we obtain 


$$
\begin{aligned}
& \varphi(r, y, t)=\lim _{\epsilon \rightarrow 0}\left[\int_{0}^{\lambda_{2}-\varepsilon} \frac{\sin \left(v_{1} t\right)(k \cosh k(y-h)+G \sinh k(y-h))}{v_{l}(k \cosh k h-G \sinh k h)} \hat{G}(k) k J_{0}(k r) d k+\right. \\
& +\int_{\lambda_{2}+\varepsilon}^{\lambda_{3}} \frac{\sinh \left(v_{2} t\right)(\cosh k(y-h)+G \sinh k(y-h))}{v_{2}(k \cosh k h-G \sinh k h)} \hat{G}(k) k J_{0}(k r) d k+ \\
& \left.+\int_{\lambda_{3}}^{\infty} \frac{\sin \left(v_{3} t\right)(\cosh k(y-h)+G \sinh k(y-h))}{v_{3}(k \cosh k h-G \sinh k h)} \hat{G}(k) k J_{0}(k r) d k\right] .
\end{aligned}
$$

Free surface depression in the case of initial axially symmetric depression and for $G h>1$ is given by

$$
\begin{aligned}
& \eta_{3}(r, t)=\lim _{\epsilon \rightarrow 0}\left[\int_{0}^{\lambda_{2}-\varepsilon} \cos \left(v_{1} t\right) \hat{G}(k) k J_{0}(k r) d k+\int_{\lambda_{2}+\varepsilon}^{\lambda_{3}} \cosh \left(v_{2} t\right) \hat{G}(k) k J_{0}(k r) d k+\right. \\
& \left.+\int_{\lambda_{3}}^{\infty} \cos \left(v_{3} t\right) \hat{G}(k) k J_{0}(k r) d k\right] .
\end{aligned}
$$

Similarly, in the case of axially symmetric impulse at the free surface and $G h>1$, the expression for depression of free surface at time $t$ is obtained as

$$
\begin{aligned}
& \eta_{4}(r, t)=\lim _{\epsilon \rightarrow 0}\left[\frac{1}{\rho} \int_{0}^{\lambda_{2}-\varepsilon} v_{1} \sin \left(v_{1} t\right) \hat{F}(k) k J_{0}(k r) d k-\frac{1}{\rho_{\lambda_{2}+\varepsilon}} \int_{3}^{\lambda_{3}} v_{2} \sinh \left(v_{2} t\right) \hat{F}(k) k J_{0}(k r) d k+\right. \\
& \left.+\frac{1}{\rho} \int_{\lambda_{3}}^{\infty} v_{3} \sin \left(v_{3} t\right) \hat{F}(k) k J_{0}(k r) d k\right] .
\end{aligned}
$$

Case C $(\boldsymbol{G h}=1)$ :

For $G h=1$ the graph of $\Omega^{2}(k)$ is shown in Fig. 4 and in this case, $\Omega^{2}(k)$ can be written as

$$
\Omega^{2}(k)=\left\{\begin{array}{l}
-\chi_{1}^{2}(k) \text { for } \varepsilon<k<\lambda_{4} \\
\chi_{2}^{2}(k) \text { for } \lambda_{4} \leq k<\infty
\end{array}\right.
$$

where $\varepsilon$ is an arbitrary small positive real number. Then by a similar procedure the form of the free surface in the case of axially symmetric depression is obtained as

$$
\eta_{5}(r, t)=\lim _{\epsilon \rightarrow 0}\left[\int_{\varepsilon}^{\lambda_{4}} \cosh \left(\chi_{1} t\right) \hat{G}(k) k J_{0}(k r) d k+\int_{\lambda_{4}}^{\infty} \cos \left(\chi_{2} t\right) \hat{G}(k) k J_{0}(k r) d k\right],
$$

and in the case of axially symmetric impulse the form of free surface is obtained as

$$
\eta_{6}(r, t)=\lim _{\epsilon \rightarrow 0}\left[-\frac{1}{\rho} \int_{\varepsilon}^{\lambda_{4}} \chi_{1} \sinh \left(\chi_{1} t\right) \hat{F}(k) k J_{0}(k r) d k+\frac{1}{\rho} \int_{\lambda_{4}}^{\infty} \chi_{2} \sin \left(\chi_{2} t\right) \hat{F}(k) k J_{0}(k r) d k\right] .
$$




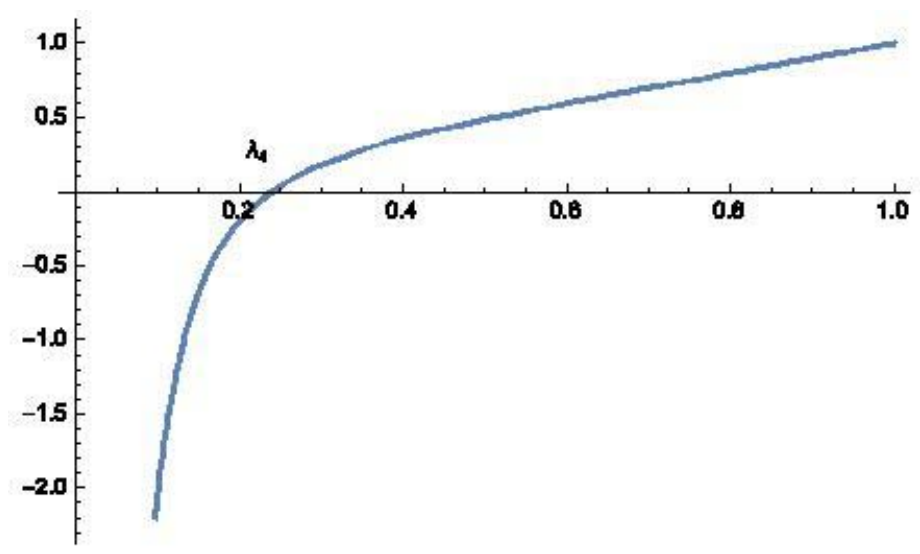

Fig.4. Graph of $\Omega^{2}(k)$ for $G h=1$.

\section{Asymptotic expansion}

We are interested in the waves after a long lapse of time and at a large distance from the origin. For this we use the method of stationary phase to evaluate the integral Eqs (3.11), (3.12) of Case A, Eqs (3.15), (3.16) of Case $\mathrm{B}$ and Eqs (3.17), (3.18) of Case $\mathrm{C}$ for large $r$ and $t$ such that $\frac{r}{t}$ is finite.

Case $A(G h<1)$ :

In particular, when the displacement is concentrated at the origin, then the initial axially symmetric depression $G(r)$ of the free surface is taken as delta function. Therefore, we can take $\hat{G}(k)=\frac{1}{2 \pi}$ and Eq.(3.11) becomes

$$
\eta_{1}(r, t)=\frac{1}{2 \pi}\left[\int_{0}^{\lambda_{1}} \cosh \left(\mu_{1} t\right) k J_{0}(k r) d k+\int_{\lambda_{1}}^{\infty} \cos \left(\mu_{2} t\right) k J_{0}(k r) d k\right] .
$$

Using the result

$$
J_{0}(k r)=\frac{2}{\pi} \int_{0}^{\frac{\pi}{2}} \cos (k r \cos \theta) d \theta
$$

and applying the method of stationary phase on $\theta$-integral we have

$$
\begin{aligned}
& \eta_{1}(r, t)=\frac{1}{(2 \pi)^{3 / 2}} \int_{0}^{\lambda_{1}}\left(\frac{k}{r}\right)^{1 / 2} \cosh \left(\mu_{1} t\right) \cos \left(k r-\frac{\pi}{4}\right) d k+ \\
& +\frac{1}{(2 \pi)^{3 / 2}} \int_{\lambda_{1}}^{\infty}\left(\frac{k}{r}\right)^{1 / 2} \cos \left(\mu_{2} t\right) \cos \left(k r-\frac{\pi}{4}\right) d k=I_{1}+I_{2} .
\end{aligned}
$$

The integral $I_{l}$ does not contribute to $\eta_{1}(r, t)$ and $I_{1}$ can be written as 


$$
I_{l}=\frac{1}{2(2 \pi)^{3 / 2}} \operatorname{Re}\left[\int_{0}^{\lambda_{l}}\left(\frac{k}{r}\right)^{1 / 2} e^{\mu_{l} t} e^{i r\left(k-\frac{\pi}{4 r}\right)} d k+\int_{0}^{\lambda_{l}}\left(\frac{k}{r}\right)^{1 / 2} e^{-\mu_{l} t} e^{i r\left(k-\frac{\pi}{4 r}\right)} d k\right] .
$$

For the first integral, we write

$$
f_{l}(k)=k-\frac{\pi}{4 r}
$$

and

$$
g_{1}(k)=\left(\frac{k}{r}\right)^{1 / 2} e^{\mu_{1} t}
$$

and $r$ is large. Now

$$
f_{1}^{\prime}(k)=1
$$

which has no zero in the range of integration.

Similarly, for the second integral we write

$$
f_{2}(k)=k-\frac{\pi}{4 r}, \quad g_{2}(k)=\left(\frac{k}{r}\right)^{1 / 2} e^{\mu_{1} t}
$$

and $r$ is large. Thus $I_{1}$ does not contribute to $\eta_{l}(r, t)$ as

$$
f_{2}^{\prime}(k)=-1
$$

which has no zero in the range of integration. For $I_{2}$ we have

$$
\begin{aligned}
& \eta_{1}(r, t)=\frac{1}{(2 \pi)^{3 / 2}} \int_{\lambda_{1}}^{\infty}\left(\frac{k}{r}\right)^{1 / 2}\left[\cos \left(\mu_{2} t+k r-\frac{\pi}{4}\right)+\cos \left(\mu_{2} t-k r+\frac{\pi}{4}\right)\right] d k= \\
& =\frac{1}{(2 \pi)^{3 / 2}} \operatorname{Re}\left[\int_{\lambda_{I}}^{\infty}\left(\frac{k}{r}\right)^{1 / 2}\left[e^{i t\left(\mu_{2}+k \frac{r}{t}-\frac{\pi}{4 t}\right)}+e^{i t\left(\mu_{2}-k \frac{r}{t}+\frac{\pi}{4 t}\right)}\right] d k\right] .
\end{aligned}
$$

In Eq.(4.2) the first integral does not have any stationary point in the range of the integration. So this integral does not contribute to $\eta_{l}(r, t)$. For the second integral let $f_{3}(k)=\mu_{2}(k)-k \frac{r}{t}+\frac{\pi}{4 t}, g_{3}(k)=\left(\frac{k}{r}\right)^{1 / 2}$ and $t$ is large. Now

$$
f_{3}^{\prime}(k)=\frac{1}{2}\left[\frac{\left(G h\left(1-\tanh ^{2} k h\right)-1\right) \mu_{2}(k)}{(k-G \tanh k h)}+\frac{\left(k^{2} h\left(1-\tanh ^{2} k h\right)+2 k \tanh k h-G\right) \mu_{2}^{-1}(k)}{(k-G \tanh k h)}\right]-\frac{r}{t} .
$$

We see that $f_{3}^{\prime}(k)$ is monotonically decreasing in $\left(\lambda_{1}, \infty\right)$ and has only one zero in the range of integration for $\frac{r}{t}>0$. Let $\alpha$ be the unique real root of 


$$
f_{3}^{\prime}(k)=0
$$

in the range of integration and $f_{3}{ }^{\prime \prime}(\alpha)$ is negative. Then

$$
\eta_{1}(r, t) \approx \frac{1}{(2 \pi)^{\frac{3}{2}}}\left(\frac{2 \pi}{t\left|f_{3}^{\prime \prime}(\alpha)\right|}\right)^{\frac{1}{2}} g_{3}(\alpha) \cos \left(t f_{3}(\alpha)-\frac{\pi}{4}\right) \text {. }
$$

Similarly, in the case of Eq.(3.12) if initial impulse is assumed to be concentrated at the origin, then $\hat{F}(k)=\frac{1}{2 \pi}$ and $\eta_{2}(r, t)$ can be written as

$$
\eta_{2}(r, t)=\frac{1}{2 \pi \rho}\left[-\int_{0}^{\lambda_{1}} \mu_{1} \sinh \left(\mu_{1} t\right) k J_{0}(k r) d k+\int_{\lambda_{1}}^{\infty} \mu_{2} \sin \left(\mu_{2} t\right) k J_{0}(k r) d k\right]=I_{3}+I_{4} .
$$

In Eq.(4.4) $I_{3}$ does not contribute to $\eta_{2}(r, t)$ and by similar reasoning for large $r$ and $t$, the asymptotic form of $\eta_{2}(r, t)$ is obtained as

$$
\eta_{2}(r, t) \approx \frac{1}{\rho(2 \pi)^{\frac{1}{2}}}\left(\frac{2 \pi}{t\left|f_{4}^{\prime \prime}(\beta)\right|}\right)^{\frac{1}{2}} g_{4}(\beta) \sin \left(t f_{4}(\beta)-\frac{\pi}{4}\right)
$$

where

$$
f_{4}(k)=\mu_{1}(k)-k \frac{r}{t}+\frac{\pi}{4 t}, \quad g_{4}(k)=\mu_{l}(k)\left(\frac{k}{r}\right)^{1 / 2},
$$

and $\beta$ is the unique real root of

$$
f_{4}^{\prime}(k)=0,
$$

in the range of integration and negative sign is taken since the sign of $f_{4}^{\prime \prime}(\beta)$ is negative.

Case B $(G h>1)$ :

For $G h>1$ if the displacement is concentrated at the origin and taking $\hat{G}(k)=\frac{1}{2 \pi}$ Eq.(3.15) becomes

$$
\begin{aligned}
& \eta_{3}(r, t)=\lim _{\epsilon \rightarrow 0}\left[\frac{1}{2 \pi} \int_{0}^{\lambda_{2}-\varepsilon} \cos \left(v_{1} t\right) k J_{0}(k r) d k+\frac{1}{2 \pi} \int_{\lambda_{2}+\varepsilon}^{\lambda_{3}} \cosh \left(v_{2} t\right) k J_{0}(k r) d k+\right. \\
& \left.+\frac{1}{2 \pi} \int_{\lambda_{3}}^{\infty} \cos \left(v_{3} t\right) k J_{0}(k r) d k\right] .
\end{aligned}
$$

In Eq.(4.6) by similar calculation the second integral does not contribute to $\eta_{3}(r, t)$ and the asymptotic form of $\eta_{3}(r, t)$ is given by 


$$
\begin{aligned}
& \eta_{3}(r, t) \approx \frac{1}{(2 \pi)^{\frac{3}{2}}}\left(\frac{\alpha_{1}}{r}\right)^{1 / 2}\left(\frac{2 \pi}{t\left|\Psi_{2}{ }^{\prime \prime}\left(\alpha_{1}\right)\right|}\right)^{\frac{1}{2}} \cos \left(t \Psi_{2}\left(\alpha_{1}\right)-\frac{\pi}{4}\right)+ \\
& +\frac{1}{(2 \pi)^{\frac{3}{2}}}\left(\frac{\alpha_{2}}{r}\right)^{1 / 2}\left(\frac{2 \pi}{t\left|\Psi_{3}{ }^{\prime \prime}\left(\alpha_{2}\right)\right|}\right)^{\frac{1}{2}} \cos \left(t \Psi_{3}\left(\alpha_{2}\right)-\frac{\pi}{4}\right)
\end{aligned}
$$

where

$$
\Psi_{2}(k)=v_{l}(k)-k \frac{r}{t}+\frac{\pi}{4 t}
$$

and $\alpha_{1}$ is the only zero of $\Psi_{2}{ }^{\prime}(k)$ in $\left(0, \lambda_{1}-\epsilon\right)$ and

$$
\Psi_{3}(k)=v_{3}(k)-k \frac{r}{t}+\frac{\pi}{4 t}
$$

and $\alpha_{2}$ is the only zero of $\Psi_{3}^{\prime}(k)$ in $\left(\lambda_{2}, \infty\right)$. Similarly, in the case of Eq.(3.16) if initial impulse is concentrated at the origin then taking $\hat{F}(k)=\frac{1}{2 \pi}, \eta_{4}(r, t)$ can be written as

$$
\begin{aligned}
& \eta_{4}(r, t)=\lim _{\epsilon \rightarrow 0}\left[\frac{1}{2 \pi \rho} \int_{0}^{\lambda_{2}-\varepsilon} v_{1} \sin \left(v_{1} t\right) k J_{0}(k r) d k-\frac{1}{2 \pi \rho} \int_{\lambda_{2}+\varepsilon}^{\lambda_{3}} v_{2} \sinh \left(v_{2} t\right) k J_{0}(k r) d k+\right. \\
& \left.+\frac{1}{2 \pi \rho} \int_{\lambda_{3}}^{\infty} v_{3} \sin \left(v_{3} t\right) k J_{0}(k r) d k\right] .
\end{aligned}
$$

The asymptotic form of $\eta_{4}(r, t)$ is given by

$$
\begin{aligned}
& \eta_{4}(r, t) \approx \frac{1}{\rho(2 \pi)^{\frac{3}{2}}} v_{l}\left(\beta_{1}\right)\left(\frac{\beta_{1}}{r}\right)^{1 / 2}\left(\frac{2 \pi}{t\left|\Psi_{4}{ }^{\prime \prime}\left(\beta_{1}\right)\right|}\right)^{\frac{1}{2}} \sin \left(t \Psi_{4}\left(\beta_{1}\right)-\frac{\pi}{4}\right)+ \\
& +\frac{1}{\rho(2 \pi)^{\frac{3}{2}}} v_{3}\left(\beta_{2}\right)\left(\frac{\beta_{2}}{r}\right)^{1 / 2}\left(\frac{2 \pi}{t\left|\Psi_{5} "\left(\beta_{2}\right)\right|}\right)^{\frac{1}{2}} \cos \left(t \Psi_{5}\left(\beta_{2}\right)-\frac{\pi}{4}\right)
\end{aligned}
$$

where

$$
\Psi_{4}(k)=v_{l}(k)-k \frac{r}{t}+\frac{\pi}{4 t}
$$

and $\beta_{1}$ is the only zero of $\Psi_{4}{ }^{\prime}(k)$ in $\left(0, \lambda_{2}-\epsilon\right)$ and

$$
\Psi_{5}(k)=v_{3}(k)-k \frac{r}{t}+\frac{\pi}{4 t}
$$

and $\beta_{2}$ is the only zero of $\Psi_{5}^{\prime}(k)$ in $\left(\lambda_{3}, \infty\right)$. 


\section{Case $\mathbf{C}(\boldsymbol{G h}=1)$ :}

Similarly, in the case of $G h=1$ the asymptotic form of $\eta_{5}(r, t)$, taking initial depression concentrated at the origin, we have

$$
\eta_{5}(r, t) \approx \frac{1}{(2 \pi)^{\frac{3}{2}}}\left(\frac{2 \pi}{t\left|f_{5}^{\prime \prime}\left(\alpha_{5}\right)\right|}\right)^{\frac{1}{2}} g_{5}\left(\alpha_{5}\right) \cos \left(t f_{5}\left(\alpha_{5}\right)-\frac{\pi}{4}\right)
$$

where

$$
f_{5}(k)=\chi_{2}(k)-k \frac{r}{t}+\frac{\pi}{4 t}, \quad g_{5}(k)=\left(\frac{k}{r}\right)^{1 / 2},
$$

and $\alpha_{5}$ is the unique positive real root of $f_{5}^{\prime}(k)=0$ in $\left(\lambda_{4}, \infty\right)$. Again, the asymptotic form of $\eta_{6}(r, t)$ taking initial impulse concentrated at the origin, we find

$$
\eta_{6}(r, t) \approx \frac{1}{\rho(2 \pi)^{\frac{1}{2}}}\left(\frac{2 \pi}{t\left|f_{6}\left(\beta_{6}\right)\right|}\right)^{\frac{1}{2}} g_{6}\left(\beta_{6}\right) \sin \left(t f_{6}\left(\beta_{6}\right)-\frac{\pi}{4}\right)
$$

where

$$
f_{6}(k)=\chi_{2}(k)-k \frac{r}{t}+\frac{\pi}{4 t}, \quad g_{6}(k)=\chi_{2}(k)\left(\frac{k}{r}\right)^{1 / 2},
$$

and $\beta_{6}$ is the unique positive real root of $f_{6}^{\prime}(k)=0$ in $\left(\lambda_{4}, \infty\right)$.

\section{Parabolic impulse}

We consider a different type of axially symmetric impulse, namely parabolic impulse distribution at the free surface given by

$$
F(r)=\left\{\begin{array}{llll}
1-\frac{1}{2}\left(\frac{r}{R}\right)^{2} & \text { for } & & r \leq \sqrt{2} R \\
0 & \text { for } & & r>\sqrt{2} R
\end{array}\right.
$$

where $R$ denotes the effective radius of initial impulse. The Hankel transform of $F(r)$ is given by

$$
\hat{F}(k)=\frac{2}{k^{2}} J_{2}(\sqrt{ } 2 R k) .
$$

For $G h<1$ the free surface depression at time $t$ for parabolic impulse distribution is given by

$$
\begin{aligned}
& \eta_{2}{ }^{\prime}(r, t)=-\frac{2}{\rho} \int_{0}^{\lambda_{1}} \frac{\mu_{1}}{k} \sinh \left(\mu_{1} t\right) J_{2}(\sqrt{ } 2 R k) J_{0}(k r) d k+ \\
& +\frac{2}{\rho} \int_{\lambda_{1}}^{\infty} \frac{\mu_{2}}{k} \sin \left(\mu_{2} t\right) J_{2}(\sqrt{ } 2 R k) J_{0}(k r) d k .
\end{aligned}
$$


For $G h>1$ the free surface depression at time $t$ for parabolic impulse distribution is given by

$$
\begin{aligned}
& \eta_{4}^{\prime}(r, t)=\lim _{\epsilon \rightarrow 0}\left[\frac{2}{\rho} \int_{0}^{\lambda_{2}-\epsilon} \frac{v_{1}}{k} \sin \left(v_{1} t\right) J_{2}(\sqrt{ } 2 R k) J_{0}(k r) d k+\right. \\
& \left.-\frac{2}{\rho} \int_{\lambda_{2}+\epsilon}^{\lambda_{3}} \frac{v_{2}}{k} \sinh \left(v_{2} t\right) J_{2}(\sqrt{ } 2 R k) J_{0}(k r) d k+\frac{2}{\rho} \int_{\lambda_{3}}^{\infty} \frac{v_{3}}{k} \sin \left(v_{3} t\right) J_{2}(\sqrt{ } 2 R k) J_{0}(k r) d k\right] .
\end{aligned}
$$

Using the result

$$
J_{2}(\sqrt{ } 2 R k)=\frac{1}{\pi} \int_{0}^{\pi} \cos (2 r-\sqrt{2 R k} \sin \tau) d \tau
$$

and

$$
J_{0}(k r)=\frac{2}{\pi} \int_{0}^{\frac{\pi}{2}} \cos (k r \cos \theta) d \theta
$$

$\eta_{2}{ }^{\prime}(r, t)$ can be written in terms of a multiple integral. This multiple integral can be evaluated approximately by the method of steepest-descent(cf. Jeffreys and Lapwood [3]). Usually, the use of the method of steepestdescent for a single integral requires a large parameter in the exponential term of the integrand. However, Jeffreys and Lapwood [3] obtained steepest-descent approximations of multiple integrals whose integrands do not have any large parameter explicitly in the exponential term. In this method one has to orient the integration paths to pass through the saddle points and the maximum contribution to the integral comes from the immediate neighbourhood of the saddle points. In the integral (4.12) the only contribution comes from the second integral and $\eta_{2}^{\prime}(r, t)$ is written in the following form

$$
\begin{aligned}
& \eta_{2}^{\prime}(r, t)=\frac{1}{2 \rho \pi^{2}} \operatorname{Im} \int_{\lambda_{1}}^{\infty} \int_{0}^{\pi} \int_{0}^{\pi} \frac{\mu_{2}}{k}\left[e^{i\left(\mu_{2} t+2 r-\sqrt{2} R k \sin \tau+k r \sin \beta\right)}+e^{i\left(\mu_{2} t-2 r+\sqrt{2} R k \sin \tau-k r \sin \beta\right)}+\right. \\
& \left.+e^{i\left(\mu_{2} t+2 r-\sqrt{2} R k \sin \tau-k r \sin \beta\right)}+e^{i\left(\mu_{2} t-2 r+\sqrt{2} R k \sin \tau+k r \sin \beta\right)}\right] d \beta d \tau d k=J_{1}+J_{2}+J_{3}+J_{4} .
\end{aligned}
$$

The integrals $J_{1}$ and $J_{4}$ do not contribute to $\eta_{2}{ }^{\prime}(r, t)$ and for $J_{2}$ we have

$$
J_{2}=\frac{1}{2 \rho \pi^{2}} \operatorname{Im} \int_{\lambda_{1}}^{\infty} \int_{0}^{\pi} \int_{0}^{\pi} G(k, \beta, \tau) \exp g(k, \beta, \tau) d \beta d \tau d k
$$

where

and

$$
G(k, \beta, \tau)=\frac{1}{k} \sqrt{\frac{k(k \tanh k h-G)}{(k-G \tanh k h)}}
$$

$$
g(k, \beta, \tau)=i\left(\mu_{2} t-2 r+\sqrt{2} R k \sin \tau-k r \sin \beta\right) .
$$

Saddle points $k_{0}, \tau_{0}, \beta_{0}$ are the solution of 


$$
\frac{\partial g}{\partial k}=\frac{\partial g}{\partial \beta}=\frac{\partial g}{\partial \tau}=0
$$

which gives $\beta_{0}=\frac{\pi}{2}, k_{0}$ is the positive root of the equation

$$
\mu_{2}^{\prime}(k)-\frac{r}{t}=-\frac{\sqrt{2\left(R^{2} k^{2}-2\right)}}{k t} \quad \text { and } \quad \tau_{0}=\cos ^{-1}\left(\frac{\sqrt{ } 2}{R k_{0}}\right) .
$$

The saddle point evaluation gives the integral to be

$$
\operatorname{Im} \frac{(i \sqrt{2 \pi})^{2}}{2 \rho \pi^{2} \sqrt{\Delta_{0}}} G_{0} \exp \left(g_{0}\right)
$$

where

$$
G_{0}=G\left(k_{0}, \beta_{0}, \tau_{0}\right) \quad \text { and } \quad g_{0}=g\left(k_{0}, \beta_{0}, \tau_{0}\right)
$$

and $\Delta_{0}$ is the value of the Hessian determinant $\Delta$ at the saddle points. Thus

$$
J_{2}=\frac{(i \sqrt{2 \pi})^{2}}{2 \rho \pi^{2}} \frac{G_{0}}{\sqrt{\Delta_{0}}} \sin \left(g\left(k_{0}, \beta_{0}, \tau_{0}\right)-\frac{\pi}{2}\right)
$$

and applying the same calculation to $J_{4}$, we obtain the final form of $\eta_{2}{ }^{\prime}(r, t)$ as,

where

$$
\eta_{2}{ }^{\prime}(r, t) \approx \frac{(i \sqrt{2 \pi})^{2}}{2 \rho \pi^{2}}\left[\frac{G\left(k_{0}, \beta_{0}, \tau_{0}\right)}{\sqrt{\Delta_{0}}} \sin \left(g\left(k_{0}, \beta_{0}, \tau_{0}\right)-\frac{\pi}{2}\right)+\frac{G\left(k_{l}, \beta_{1}, \tau_{l}\right)}{\sqrt{\Delta_{l}}} \sin \left(g_{1}\left(k_{1}, \beta_{1}, \tau_{l}\right)\right)\right]
$$

and

$$
G(k, \beta, \tau)=\frac{1}{k} \sqrt{\frac{k(k \tanh k h-G)}{(k-G \tanh k h)}}, \quad \Delta_{0}=\Delta\left(k_{0}, \beta_{0}, \tau_{0}\right)
$$

$$
\Delta(k, \beta, \tau)=\sqrt{2} t \mu^{\prime \prime}{ }_{2}(k) r R k^{2} \sin \tau+2 R^{2} r k \cos ^{2} \tau
$$

and $k_{0}, \beta_{0}, \tau_{0}$ are the saddle points in $\beta, \tau, k$ - plane for the integral $J_{2}$ and

$$
\begin{aligned}
& g_{l}(k, \beta, \tau)=i\left(\mu_{2} t+2 r-\sqrt{2} R k \sin \tau-k r \sin \beta\right), \\
& \Delta_{l}=\Delta^{\prime}\left(k_{1}, \beta_{1}, \tau_{l}\right) \quad \text { and } \quad \Delta^{\prime}(k, \beta, \tau)=-\sqrt{2} t \mu^{\prime \prime}{ }_{2}(k) r R k^{2} \sin \tau+2 R^{2} r k \cos ^{2} \tau
\end{aligned}
$$

and $k_{1}, \beta_{1}, \tau_{1}$ are the saddle points for the integral $J_{3}$.

A similar process is applied to the integral (4.11) for the case $G h>1$ and in this case the only contribution comes from the third integral and the final result is obtained as

$$
\eta_{4}^{\prime}(r, t) \approx \frac{(i \sqrt{2 \pi})^{2}}{2 \rho \pi^{2}}\left[\frac{G^{\prime}\left(k_{0}^{\prime}, \beta_{0}^{\prime}, \tau_{0}^{\prime}\right)}{\sqrt{\Delta_{0}^{\prime}}} \sin \left(g_{2}\left(k_{0}^{\prime}, \beta_{0}^{\prime}, \tau_{0}^{\prime}\right)-\frac{\pi}{2}\right)+\frac{G^{\prime}\left(k_{l}^{\prime}, \beta_{1}^{\prime}, \tau_{l}^{\prime}\right)}{\sqrt{\Delta_{l}^{\prime}}} \sin \left(g_{3}\left(k_{l}^{\prime}, \beta_{l}^{\prime}, \tau_{l}^{\prime}\right)\right)\right]
$$


where

$$
G^{\prime}(k, \beta, \tau)=\frac{v_{3}(k)}{k}, \quad \Delta_{0}^{\prime}=\Delta^{\prime \prime}\left(k_{0}{ }^{\prime}, \beta_{0}{ }^{\prime}, \tau_{0}^{\prime}\right)
$$

and

$$
\begin{aligned}
& \Delta^{\prime \prime}(k, \beta, \tau)=\sqrt{2} t v^{\prime \prime}(k) r R k^{2} \sin \tau+2 R^{2} r k \cos ^{2} \tau, \\
& g_{2}(k, \beta, \tau)=i\left(v_{3}(k) t-2 r+\sqrt{2} R k \sin \tau-k r \sin \beta\right)
\end{aligned}
$$

and $k_{0}{ }^{\prime}, \beta_{0}{ }^{\prime}, \tau_{0}{ }^{\prime}$ are the saddle points in $\beta, \tau, k$ - plane and

$$
\Delta_{l}^{\prime}=\Delta^{\prime \prime \prime}\left(k_{l}^{\prime}, \beta_{l}^{\prime}, \tau_{l}^{\prime}\right) \quad \text { and } \quad \Delta^{\prime \prime \prime}(k, \beta, \tau)=-\sqrt{2} t v^{\prime \prime}{ }_{3}(k) r R k^{2} \sin \tau+2 R^{2} r k \cos ^{2} \tau
$$

and

$$
g_{3}(k, \beta, \tau)=i\left(v_{3}(k) t+2 r-\sqrt{2} R k \sin \tau-k r \sin \beta\right)
$$

and $k_{l}^{\prime}, \beta_{l}^{\prime}, \tau_{l}^{\prime}$ are the saddle points in $\beta, \tau, k$ - plane.

\section{Numerical results}

Case A $(\boldsymbol{G h}<1)$ :

To study the form of the free surface due to initial axisymmetric surface disturbance in water with a porous bottom, the non dimensional asymptotic form of $\eta_{l}(r, t)$ is depicted graphically against $r$ for fixed time and against $t$ for a fixed distance from the origin in a number of figures. To visualize the nature of the wave motion due to prescribed initial axially symmetric depression at the free surface, $\eta_{l}(r, t)$ is plotted in Fig.5 against $t$ for fixed $r=250$ and $t$ ranging from 220 to 280 and porosity parameter $G h=0,0.6,0.9$. Also $\eta_{l}(r, t)$ is plotted in Fig.6 for fixed time and variable distance from the origin. In Fig.6 $\eta_{l}(r, t)$ is plotted for fixed $t=150$ and $r$ ranging from 220 to 280 and the porosity parameter $G h=0,0.6,0.9$. From Fig.5 it is observed that as $t$ increases, the amplitude of wave motion increases and from Fig.6 it is observed that as $r$ increases the amplitude of wave motion decreases.

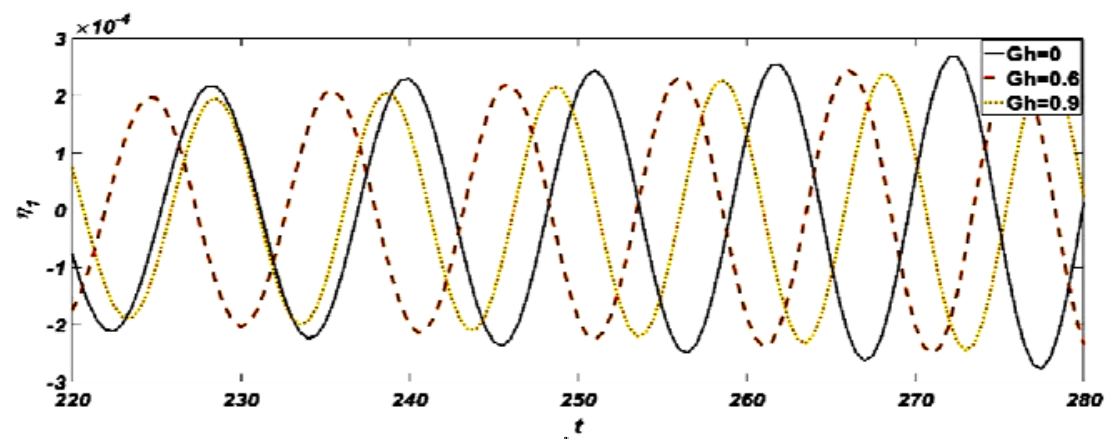

Fig.5. Wave motion due to initial axially symmetric depression for a fixed distance $r=250$. 


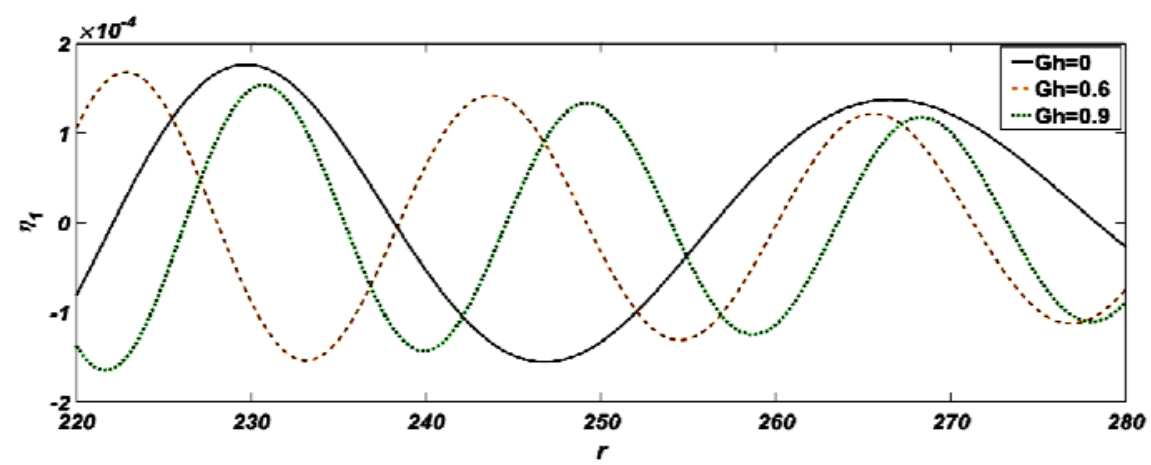

Fig.6. Wave motion due to initial axially symmetric depression for fixed time $t=150$.

Similarly $\eta_{2}(r, t)$ obtained from Eq.(4.5) due to an initial disturbance in the form of an impulse concentrated at the origin, is plotted in Figs 7 and 8. In Fig.7 $\eta_{2}(r, t)$ is plotted against $t$ for fixed $r=250$ and $t$ ranging from 220 to 280 and porosity parameter $G h=0,0.6,0.9$. From Fig. 7 it is observed that the amplitude of the wave profile increases as time increases. In Fig. $8 \eta_{2}(r, t)$ is plotted against $r$ for fixed $t=150$ and $r$ ranging from 220 to 280 and porosity parameter $G h=0,0.6,0.9$. From Fig. 8 it is observed that the amplitude of the wave profile decreases as distance increases.

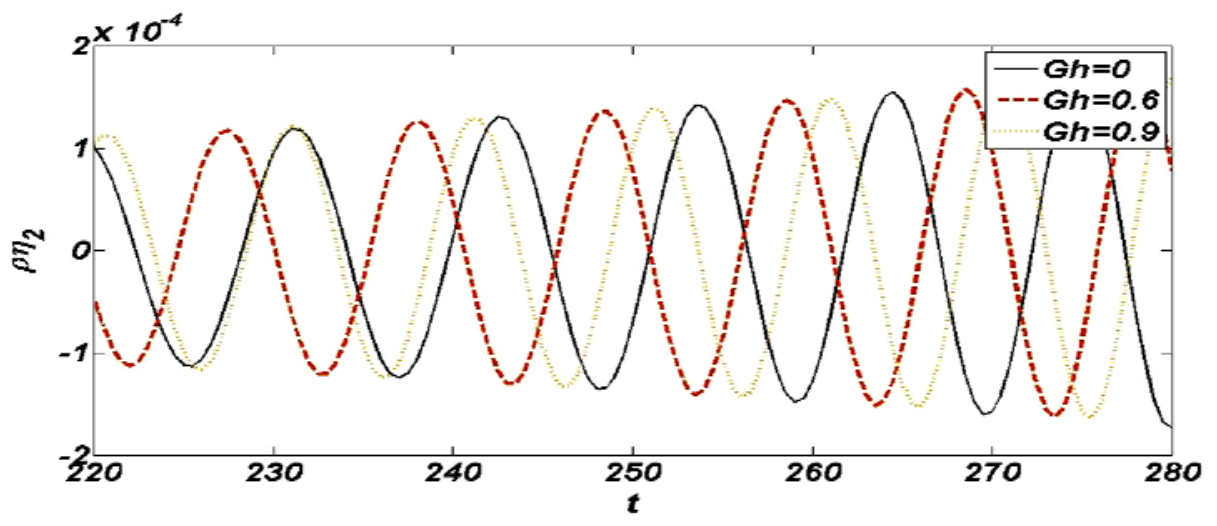

Fig.7. Wave motion due to initial axially symmetric impulse for a fixed distance $r=250$.

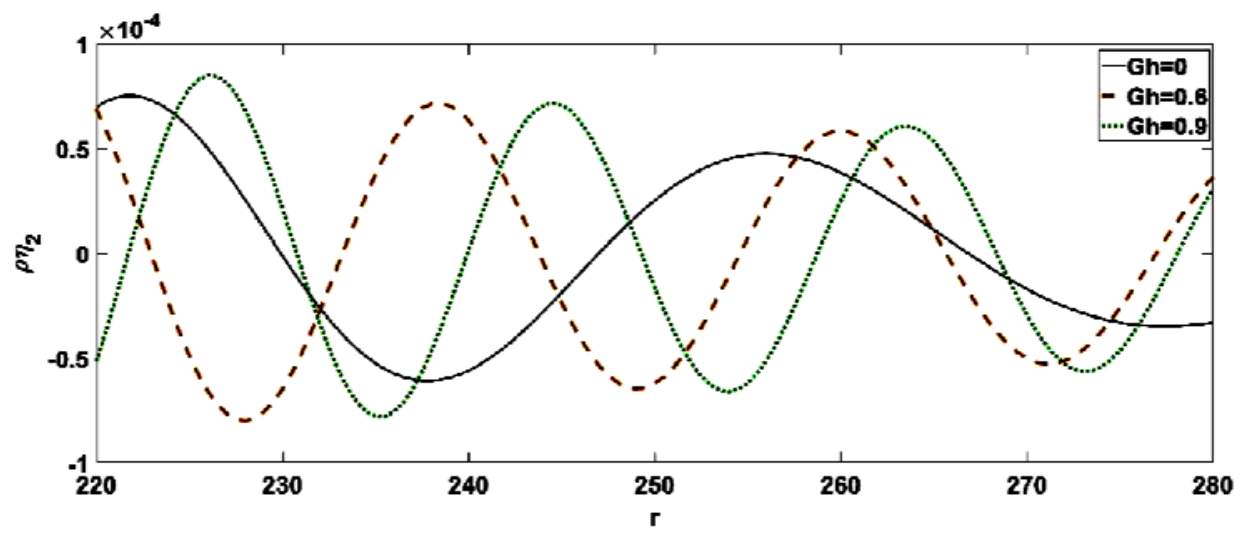

Fig.8. Wave motion due to initial axially symmetric impulse for fixed time $t=150$. 


\section{Case $B$ and $C(G h \geq 1)$ :}

For the case $G h \geq 1, \eta_{3}(r, t)$ and $\eta_{5}(r, t)$ obtained from Eqs (4.7) and (4.10) in the case of initial axially symmetric depression concentrated at the origin are plotted in Fig.9 for fixed $r=250$ and $t$ ranging from 220 to 280 and $G h=1,1.25,1.75$.

Similarly, $\eta_{3}(r, t)$ and $\eta_{5}(r, t)$ are plotted in Fig.10 for fixed time $t=150$ and $r$ ranging from 220 to 280 and $G h=1,1.25,1.75$.

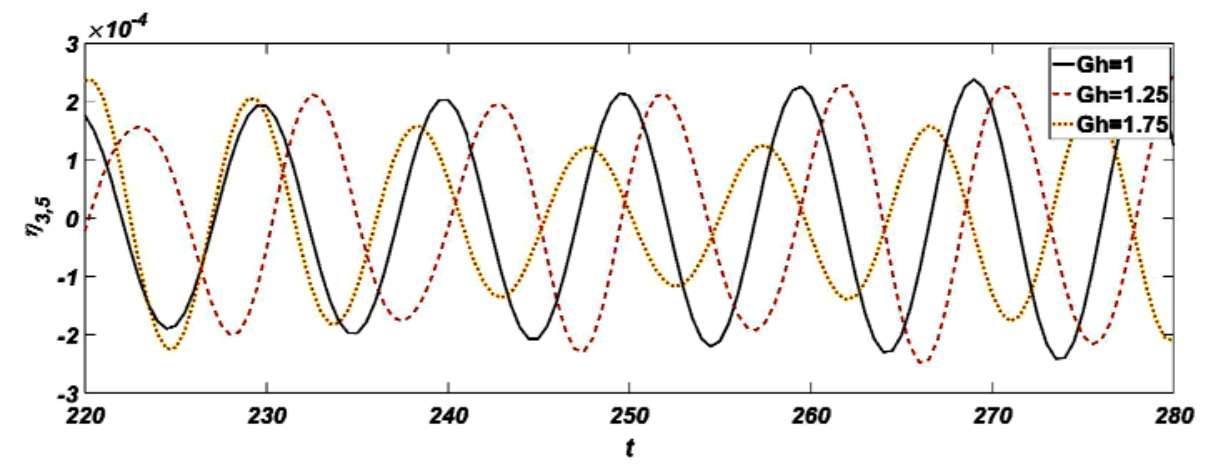

Fig.9. Wave motion due to initial axially symmetric depression for a fixed distance $r=250$.

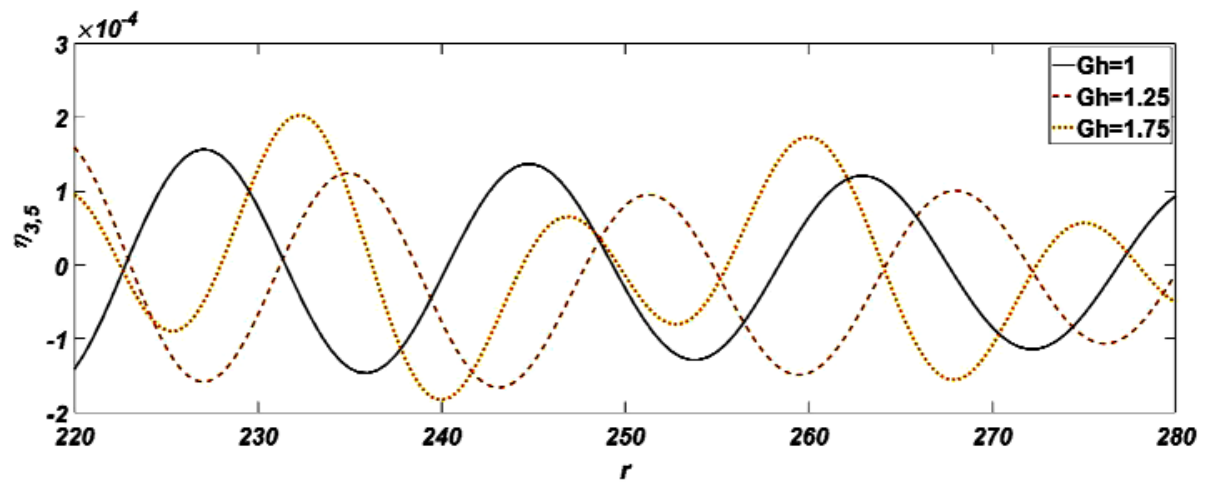

Fig.10. Wave motion due to initial axially symmetric depression for fixed time $t=150$.

Similarly, $\rho \eta_{4}(r, t)$ and $\rho \eta_{6}(r, t)$ obtained from Eqs (4.9) and (4.11) in the case of impulse concentrated at origin are plotted in Figs 11 and 12. In the Fig.11 $\rho \eta_{4}(r, t)$ and $\rho \eta_{6}(r, t)$ are plotted against $t$ for fixed $r=250$ and $t$ ranging from 220 to 280 and $G h=1,1.25,1.75$. In Fig.12 $\rho \eta_{4}(r, t)$ and $\rho \eta_{6}(r, t)$ are plotted against $r$ for fixed $t=150$ and $r$ ranging from 220 to 280 and $G h=1,1.25,1.75$. From Fig.9 to 12 it is observed that as the porosity parameter increases the amplitude of wave motion does not behave in any specific manner.

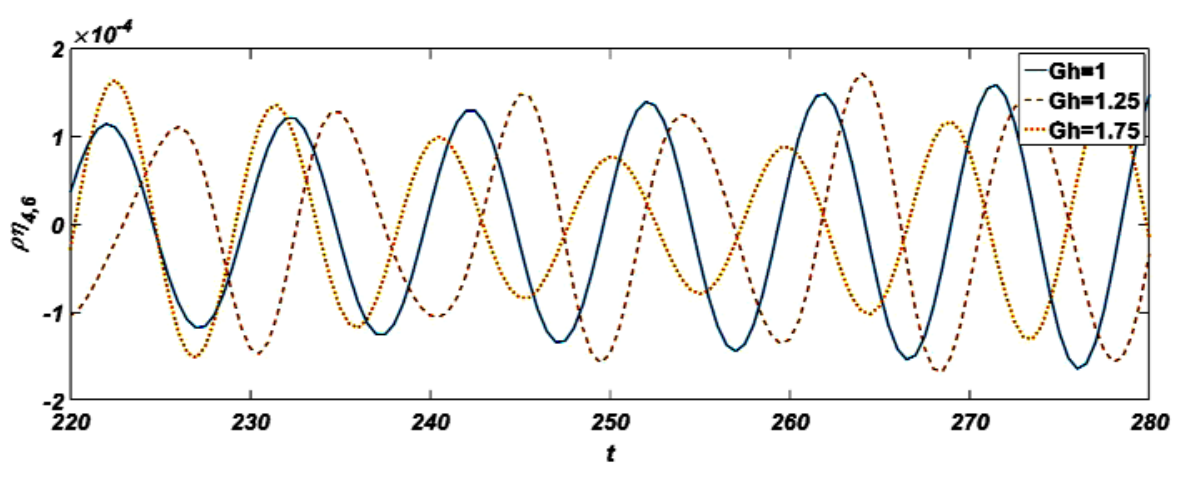

Fig.11. Wave motion due to initial axially symmetric impulse for a fixed distance $r=250$ and $G h \geq 1$. 


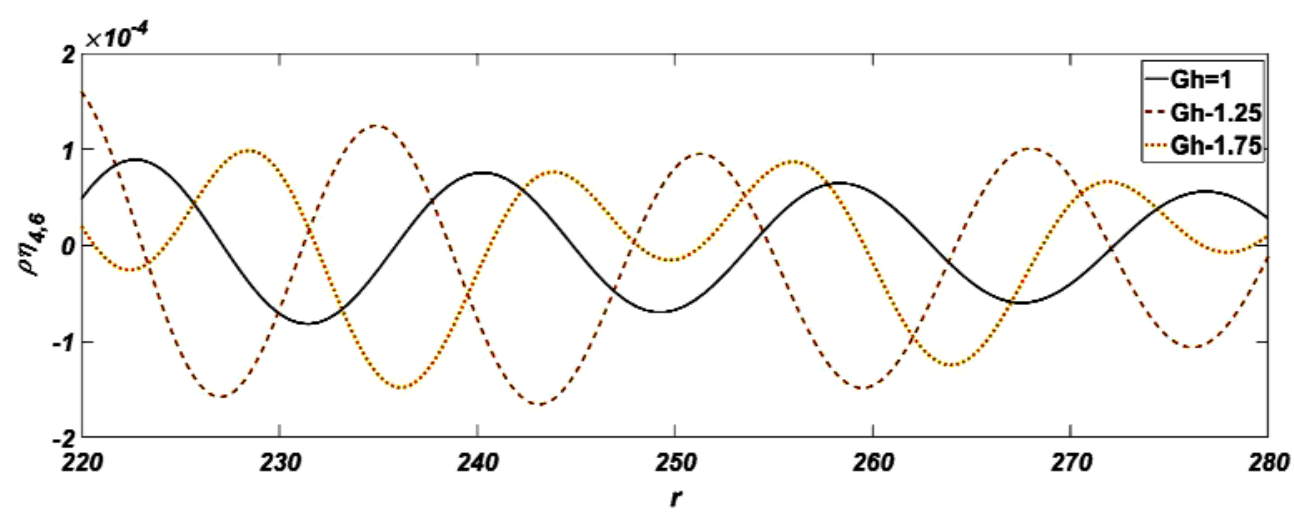

Fig.12. Wave motion due to initial axially symmetric impulse for fixed time $t=150$ and $G h \geq 1$.

\section{Parabolic Impulse :}

For the parabolic impulse distribution the form of the free surface elevation in the case of $G h<1$ given in Eq.(4.14) is plotted in Figs 13 and 14. In Fig.13 $\rho \eta_{2}{ }^{\prime}(r, t)$ is plotted against $t$ for fixed $r=250$ and $t$ ranging from 200 to 280 and $G h=0.2,0.6,0.9, R=10$. In Fig.14 $\rho \eta_{2}{ }^{\prime}(r, t)$ is plotted against $r$ for fixed $t=150$ and $r$ ranging from 220 to 280 and $G h=0.2,0.6,0.9, R=10$. Similarly, $\rho \eta_{4}{ }^{\prime}(r, t)$ obtained from Eq.(4.15) is plotted in Figs 15 and 16.

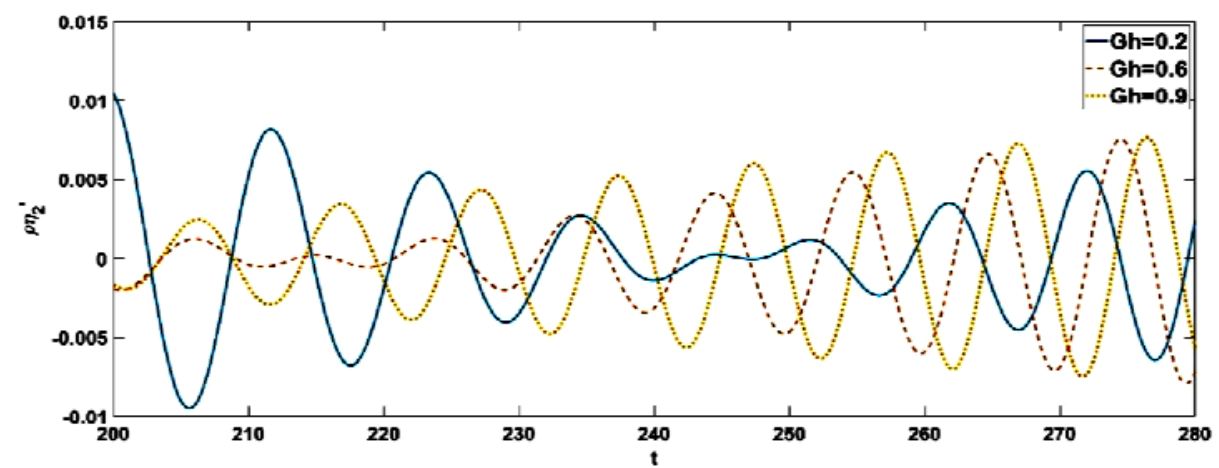

Fig.13. Wave motion due to parabolic impulse for $G h<1$ and for a fixed distance $r=250$.

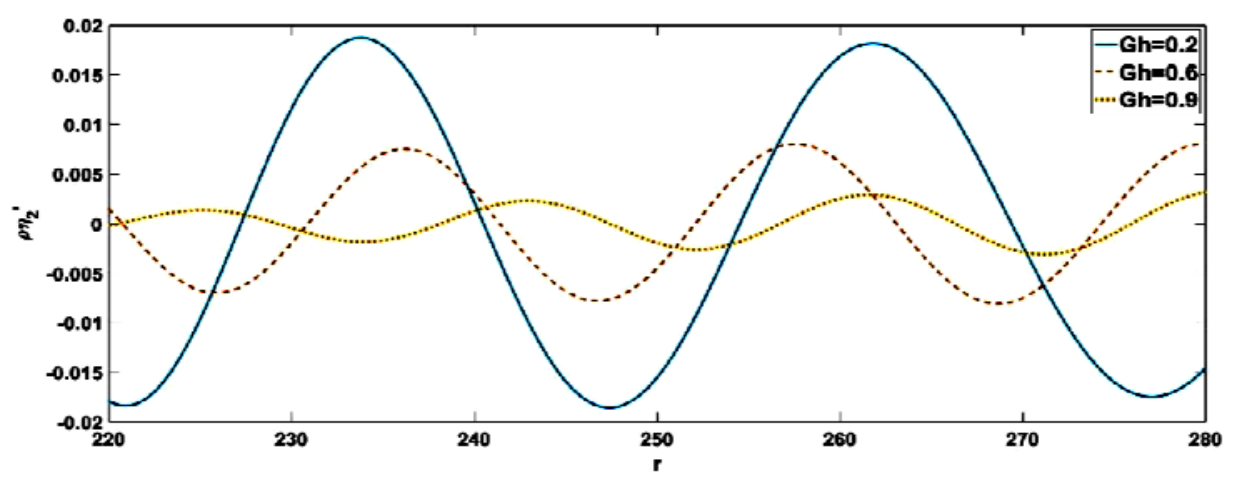

Fig.14. Wave motion due to parabolic impulse for $G h<1$ and for fixed time $t=150$. 


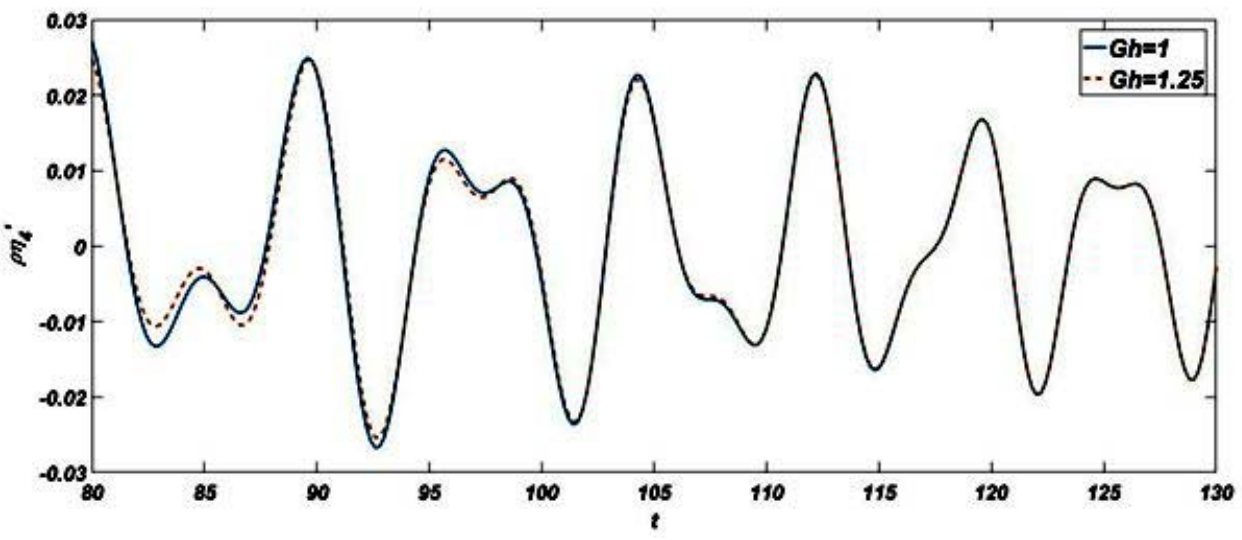

Fig.15. Wave motion due to parabolic impulse for $G h \geq 1$ and for a fixed distance $r=50$.

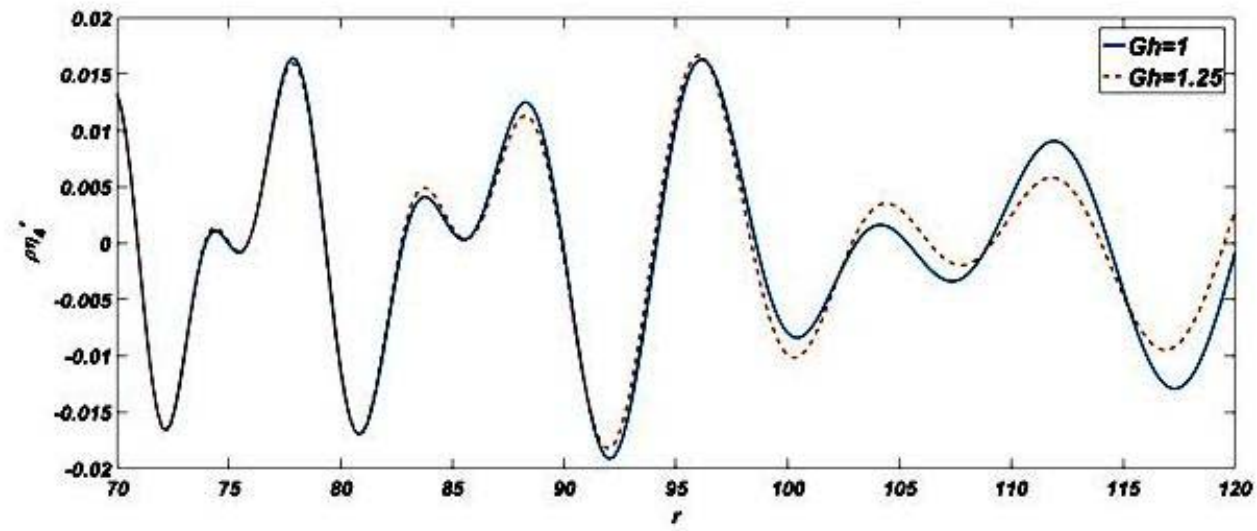

Fig.16. Wave motion due to parabolic impulse for $G h \geq 1$ and for fixed time $t=150$.

In Fig.15 $\rho \eta_{4}{ }^{\prime}(r, t)$ is plotted against $t$ for fixed $r=50$ and $t$ ranging from 80 to 130 and $G h=1,1.25, R=10$. In Fig.16 $\rho \eta_{4}{ }^{\prime}(r, t)$ is plotted against $r$ for fixed $t=150$ and $r$ ranging from 70 to 120 and $G h=1,1.25, R=10$.

\section{Conclusion}

Generation of surface waves in water with a porous bottom is considered here due to various types of prescribed initial axisymmetric disturbances at the free surface. The initial disturbance on the free surface is taken to be concentrated at the origin for the first two cases and in the form of a parabolic impulse distribution at the free surface as in Krenzer Keller [4] for the third case. The fluid bottom is taken to be porous and the porosity parameter $G$ to be real and the cases when $G h<1$ and $G h \geq 1$ and $h$ being the finite depth of water are considered. It is observed that when the bottom is rigid, the integral form of free surface reduces to the form given in Kranzer and Keller [4] for the case of parabolic impulse distribution at the free surface.

\section{Acknowledgement}

This work is carried out under CSIR research project No. 25(0253)/16/EMR-II. 


\section{Nomenclature}

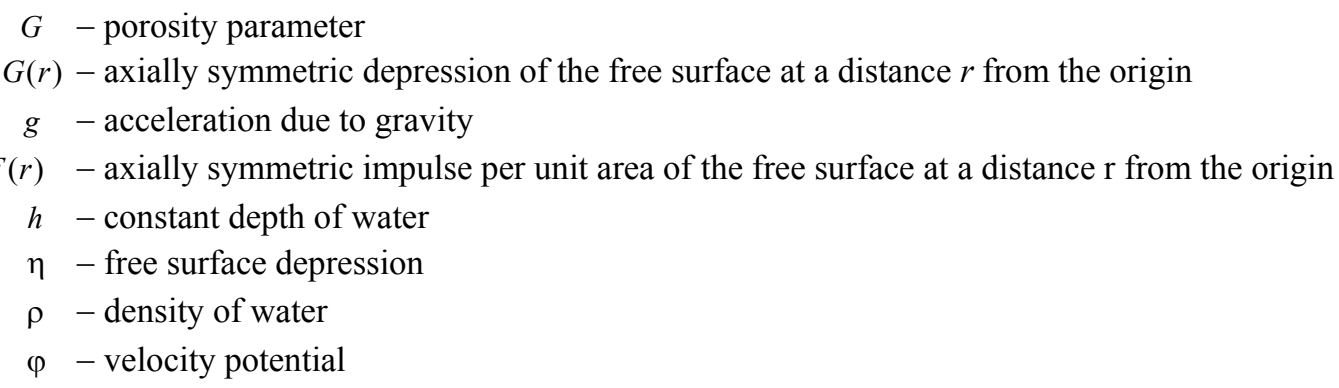

\section{References}

[1] Lamb H. (1945): Hydrodynamics. - New York: Dover.

[2] Stoker J.J. (1957): Water Waves the Mathematical Theory with Applications. - New York: Interscience Publishers.

[3] Jeffreys H. and Lapwood E.R. (1957): The reflexion of a pulse within a sphere. - Proc. Roy. Soc. A London, vol.241, pp.455-479.

[4] Kranzer H.C. and Keller J.B. (1959): Water waves produced by explosions. - J. Appl. Phys., vol.30, pp.398-407.

[5] Mandal B.N. and Mukherjee S. (1989): Water waves generated at an inertial surface by an axisymmetric initial surface disturbance. - Int. J. Math. Educ. Sci. Tech., vol.20, pp.743-747.

[6] Maiti P. and Mandal B.N. (2005): Water waves generated due to initial axisymmetric disturbances in water with an ice-cover. - Arch. Appl. Mech., vol.74, pp.629-636.

[7] Martha S.C., Bora S.N. and Chakrabarti A. (2007): Oblique water wave scattering by small undulation on a porous sea bed. - Appl. Ocean. Res., vol.29, pp.86-90.

[8] Maiti P. and Mandal B.N. (2014): Water wave scattering by an elastic plate floating in an ocean with a porous bed. - Appl. Ocean. Res., vol.47, pp.73-84.

Received: June 15, 2018

Revised: November 7, 2018 